\title{
The Omega-3 Fatty Acid Docosahexaenoic Acid Modulates Inflammatory Mediator Release in Human Alveolar Cells Exposed to Bronchoalveolar Lavage Fluid of ARDS Patients
}

\author{
Paolo Cotogni, ${ }^{1}$ Antonella Trombetta, ${ }^{2}$ Giuliana Muzio, ${ }^{3}$ \\ Marina Maggiora, ${ }^{3}$ and Rosa Angela Canuto ${ }^{3}$ \\ ${ }^{1}$ Anesthesiology and Intensive Care, Department of Medicine, S. Giovanni Battista Hospital, University of Turin, \\ Via A.M. Dogliotti 14, 10126 Turin, Italy \\ ${ }^{2}$ Department of Medical Sciences, University of Turin, Via A.M. Dogliotti 14, 10126 Turin, Italy \\ ${ }^{3}$ Department of Experimental Medicine and Oncology, University of Turin, Corso Raffaello 30, 10125 Turin, Italy \\ Correspondence should be addressed to Paolo Cotogni; paolo.cotogni@unito.it
}

Received 12 October 2014; Accepted 28 January 2015

Academic Editor: Karsten Weylandt

Copyright (C) 2015 Paolo Cotogni et al. This is an open access article distributed under the Creative Commons Attribution License, which permits unrestricted use, distribution, and reproduction in any medium, provided the original work is properly cited.

\begin{abstract}
Background. This study investigated whether the $1: 2 \omega-3 / \omega-6$ ratio may reduce proinflammatory response in human alveolar cells (A549) exposed to an ex vivo inflammatory stimulus (bronchoalveolar lavage fluid (BALF) of acute respiratory distress syndrome (ARDS) patients). Methods. We exposed A549 cells to the BALF collected from 12 ARDS patients. After 18 hours, fatty acids (FA) were added as docosahexaenoic acid (DHA, $\omega-3)$ and arachidonic acid (AA, $\omega-6)$ in two ratios (1:2 or 1:7). 24 hours later, in culture supernatants were evaluated cytokines (TNF- $\alpha$, IL-6, IL-8, and IL-10) and prostaglandins (PGE 2 and PGE 3 ) release. The FA percentage content in A549 membrane phospholipids, content of COX-2, level of PPAR $\gamma$, and NF- $\kappa$ B binding activity were determined. Results. The 1:2 DHA/AA ratio reversed the baseline predominance of $\omega-6$ over $\omega-3$ in the cell membranes $(P<0.001)$. The proinflammatory cytokine release was reduced by the $1: 2$ ratio $(P<0.01$ to $<0.001)$ but was increased by the $1: 7$ ratio $(P<0.01)$. The $1: 2$ ratio reduced COX-2 and $\mathrm{PGE}_{2}(P<0.001)$ as well as NF- $\kappa \mathrm{B}$ translocation into the nucleus $(P<0.01)$, while it increased activation of PPAR $\gamma$ and IL-10 release $(P<0.001)$. Conclusion. This study demonstrated that shifting the FA supply from $\omega-6$ to $\omega-3$ decreased proinflammatory mediator release in human alveolar cells exposed to BALF of ARDS patients.
\end{abstract}

\section{Introduction}

Acute respiratory distress syndrome (ARDS) is a form of acute diffuse lung injury associated with a predisposing risk factor, characterized by inflammation leading to increased pulmonary vascular permeability and loss of aerated lung tissue [1]. According to the Berlin definition, the acute phase of this syndrome is manifested by the early onset of respiratory failure (within 1 week of a known clinical insult or new/worsening respiratory symptoms) [2]. The main characteristic of the clinical syndrome is hypoxemia; specifically, each subcategory of ARDS (mild, moderate, and severe) is defined by mutually exclusive ranges of the ratio between partial pressure of arterial oxygen $\left(\mathrm{PaO}_{2}\right)$ and fraction of inspired oxygen $\left(\mathrm{FiO}_{2}\right)\left(200 \mathrm{~mm} \mathrm{Hg}<\mathrm{PaO}_{2} / \mathrm{FIO}_{2} \leq 300 \mathrm{~mm} \mathrm{Hg}\right.$,
$100 \mathrm{~mm} \mathrm{Hg}<\mathrm{PaO}_{2} / \mathrm{FIO}_{2} \leq 200 \mathrm{~mm} \mathrm{Hg}$, and $\mathrm{PaO}_{2} / \mathrm{FIO}_{2} \leq$ $100 \mathrm{~mm} \mathrm{Hg}$, resp.) [2]. Mortality from severe ARDS in the 1970 s was as high as $85-90 \%$ but, from 2000 , it decreased to $20-40 \%$ [3].

In the ARDS early phase, the alveolar space is characterized by alveolar infiltration with neutrophils and macrophages, and both are able to release inflammatory cytokines with an accumulation of both proinflammatory and anti-inflammatory cytokines $[4,5]$. Many cytokines were detected at elevated levels in bronchoalveolar lavage fluid (BALF) in patients with ARDS, that is, tumor necrosis factor (TNF)- $\alpha$, interleukin (IL)-1 $\beta$, IL-6, and IL-8. The key role of a hyperinflammatory response, mainly characterized by the overproduction of proinflammatory cytokines, in the progression of the lung damage is well-documented $[6,7]$. 
Moreover, many studies reported an increased mortality in patients who have elevated proinflammatory cytokine concentrations (TNF- $\alpha$, IL-6, and IL-8) in the BALF at the onset of ARDS or persistent increased concentrations $[8,9]$. Similarly, low levels of anti-inflammatory cytokines (i.e., IL10 and IL-1 receptor antagonist) in the BALF in the ARDS early phase are associated with an increased mortality [10, 11]. Thus, the balance between proinflammatory and antiinflammatory cytokines is of greater importance because the degree of cytokine imbalance is a contributing element to ARDS severity [12].

Over the last years, there has been an improving understanding of polyunsaturated fatty acid (PUFA) pathophysiology and several mechanisms for the interaction between PUFAs and inflammation or immune response have been demonstrated [13-16]. Indeed, after PUFA supply (diet or enteral and parenteral administration), many cell properties and related functions are modified, mainly the inflammatory and immunity responses [13]. Briefly, omega- ( $\omega-) 3$ PUFAs are more regarded as anti-inflammatory agents, whereas $\omega$ 6 PUFAs are regarded as proinflammatory agents. Recently, it was speculated that $\omega-3$ PUFAs may be involved in the resolution of inflammation [14].

The discovered ability of $\omega-3$ PUFAs to downregulate several different responses of the inflammatory process had suggested that these PUFAs might be used not exclusively as nutrients but mainly as pharmacological agents. Since the 1990s, many editorials have stressed the possibility to modulate the inflammatory response in acute lung injury (ALI) or ARDS patients using $\omega$-3 PUFAs as drugs (the socalled "pharmaconutrition") [17-19]. Subsequently, a great number of studies with cell or animal models have been carried out with the aim to demonstrate the efficacy of fish oil or their main active components (i.e., eicosapentaenoic acid (EPA) and docosahexaenoic acid (DHA)) in modifying the inflammatory responses [20-22]. Likewise, there have been several randomized controlled trials of enteral [23-28] or parenteral $[5,29-31]$ administration of fish oil-enriched nutrition formulas in mechanically ventilated patients with ALI, ARDS, or sepsis.

In a previous study [32], we demonstrated that shifting the PUFA supply from $\omega-6$ PUFA (i.e., arachidonic acid (AA)) to $\omega$-3 PUFA (i.e., DHA) significantly reduced the release of proinflammatory cytokines in human alveolar cells undergoing lipopolysaccharide (LPS) challenge. Moreover, we found that, in the presence of a DHA/AA ratio with a predominance of $\mathrm{AA}$, there was a cytokine balance more oriented towards a proinflammatory response than with LPS alone. The aim of this study was to investigate whether in human alveolar cells the 1:2 DHA/AA ratio was effective in reducing proinflammatory response induced by an ex vivo inflammatory stimulus such as BALF of ARDS patients.

\section{Materials and Methods}

2.1. Bronchoalveolar Lavage Fluid Collection. The BALF was collected from ARDS patients-requiring mechanical ventilation and BAL for clinical purposes-within 24 hours after endotracheal intubation and stored at $-80^{\circ} \mathrm{C}$ as previously described [33]. The selection of ARDS patients was carried out as previously described [34]. The institutional review board approved the study.

2.2. Cell Culture and Treatment. The human lung carcinoma cell line A549 (ATCC, Rockville, MD, USA) was used. A549 are alveolar epithelial cells with type II pneumocyte properties. The A549 were cultured in HAM-F12 K medium (Sigma-Aldrich, St Louis, MO, USA) and treated as previously described [35].

BALF was added 24 hours after seeding, while LPS $(400 \mu \mathrm{g} / \mathrm{mL})$ was used as positive control. After 18 hours, A549 cells were treated with $50 \mu \mathrm{M}$ of $1: 2 \mathrm{DHA} / \mathrm{AA}$ ratio (DHA $17 \mu \mathrm{M}$ plus AA $33 \mu \mathrm{M}$ ) or $50 \mu \mathrm{M}$ of $1: 7 \mathrm{DHA} / \mathrm{AA}$ ratio (DHA $6.5 \mu \mathrm{M}$ plus AA $43.5 \mu \mathrm{M}$ ) for 24 hours. PUFAs and LPS from Escherichia coli 055:B5 were obtained from SigmaAldrich.

2.3. ELISA. At the experimental end point (i.e., after 24 hours), cytokine contents were measured in BALF (i.e., IL$1 \beta$, TNF- $\alpha$, IL- 6 , and IL- 8 ) and in culture supernatants (i.e., TNF- $\alpha$, IL-6, IL-8, and IL-10) via enzyme-linked immunosorbent assay (ELISA). Cytokine kits were purchased from Euroclone (Paignton-Devon, UK). Prostaglandins $\mathrm{E}_{2}$ and $\mathrm{E}_{3}\left(\mathrm{PGE}_{2}\right.$ and $\left.\mathrm{PGE}_{3}\right)$ release was measured in culture supernatants via ELISA. Prostaglandin kits were purchased from MyBioSource (San Diego, CA, USA). The assays were performed according to the manufacturer's instructions.

2.4. Fatty Acid Percentage Content in Membrane Phospholipids. At the experimental end point, the fatty acid (FA) percentage content was determined as previously described [36]. Briefly, total lipids were extracted by the method of Folch et al. [37] and separated by thin-layer chromatography using $\mathrm{n}$-heptane: isopropylether: formic acid $(90: 60: 3)$ as solvent. Phospholipids bands (deposition line) were scraped, extracted, and used for FA determination. FA methyl esters were prepared following the method of Metcalfe et al. [38] and separated by gas-liquid chromatography (CP 9002 Chrompack International B.V., Middelburg, Netherlands). Internal standard (methyl heptadecanoate) was added to each preparation to determine recovery.

\subsection{Preparation of Total Cell Lysate for Western Blot Analysis.} Total cells lysates were obtained as previously described [35]. Briefly, cells were sonicated in HCMF buffer containing $1 \%$ Triton, $0.1 \%$ SDS, $2 \mathrm{mM}$ Calcium Chloride $\left(\mathrm{CaCl}_{2}\right), 100 \mu \mathrm{g} / \mathrm{mL}$ phenylmethylsulfonyl fluoride (PMSF), and $1 \mu \mathrm{g} / \mathrm{mL}$ leupeptin at an intermediate setting (output $\cong$ 3) using a Branson Sonifier 250 (VWR Scientific, OH, USA). Lysates were cooled on ice for 3-5 $\mathrm{min}$ and the sonicatingcooling cycle was repeated for a total of 3 cycles.

2.6. Preparation of Cytoplasmic and Nuclear Extracts for Western Blot Analysis. At the experimental end point, culture media were collected and stored at $-80^{\circ} \mathrm{C}$ until cytokine concentration evaluation with ELISA and cells were lysed for cytoplasmic and nuclear protein fractions extraction. Briefly, cells were lysed into buffer A (10 mM HEPES pH 7.9, 10 mM 


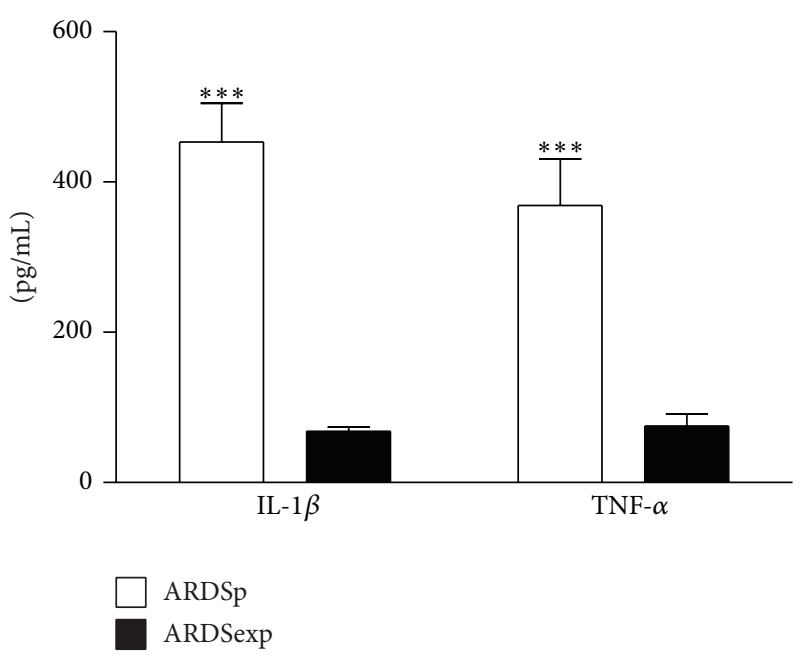

(a)

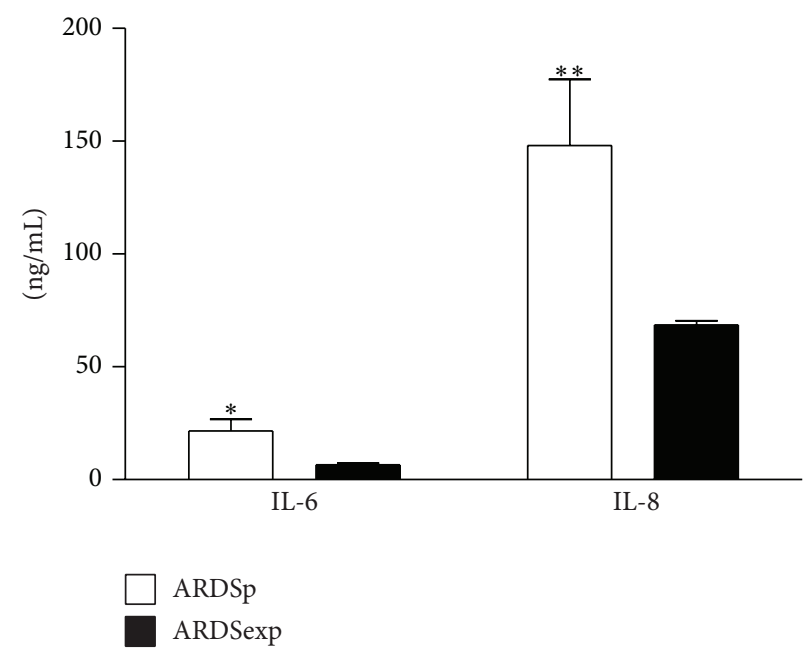

(b)

FIGURE 1: BALF cytokine pattern in ARDS. (a) IL-1 $\beta$ and TNF- $\alpha$ proinflammatory cytokine content in BALF collected from pulmonary (ARDSp, white bars) and extrapulmonary (ARDSexp, black bars) ARDS patients. (b) IL-6 and IL-8 cytokine content in BALF collected from pulmonary (ARDSp, white bars) and extrapulmonary (ARDSexp, black bars) ARDS patients. The results are expressed as picograms (pg) or nanograms (ng) of cytokines per $\mathrm{mL}$ as indicated. Data are presented as mean \pm standard deviation of 12 independent determinations $(n=7$ ARDSp and $n=5$ ARDSexp). BALF, bronchoalveolar lavage fluid; ARDS, acute respiratory distress syndrome; IL, interleukin; TNF, tumor necrosis factor. ${ }^{*} P<0.05$ ARDSp versus ARDSexp. ${ }^{* *} P<0.01$ ARDSp versus ARDSexp. ${ }^{* * *} P<0.001$ ARDSp versus ARDSexp.

$\mathrm{KCl}, 0.1 \mathrm{mM}$ EDTA) added with $1 \mathrm{mM}$ DTT, 0.5 mM PMSF, $5 \mu \mathrm{L}$ of $10 \mu \mathrm{g} / \mu \mathrm{L}$ of aprotinin, leupeptin, and pepstatin A and $40 \mu \mathrm{L} / \mathrm{mL}$ of IGEPAL $10 \%$. Cell lysates were centrifuged with a Microfuge at $15,000 \mathrm{rpm} \times 3 \mathrm{~min}$ at $4^{\circ} \mathrm{C}$. Supernatants cytoplasmic fractions were collected and stored at $-80^{\circ} \mathrm{C}$ until use. Nuclei pellets were lysated, for $2 \mathrm{~h}$ at $4^{\circ} \mathrm{C}$, in buffer B (20 mM HEPES, pH 7.9, $0.4 \mathrm{M} \mathrm{NaCl}, 1 \mathrm{mM}$ EDTA, and $10 \%$ glycerol) added with $1 \mathrm{mM} \mathrm{DTT}, 0.5 \mathrm{mM}$ PMSF, $5 \mu \mathrm{L}$ of $10 \mu \mathrm{g} / \mu \mathrm{L}$ of aprotinin, leupeptin, and pepstatin A. Nuclei lysates were centrifuged with a Microfuge at $15,000 \mathrm{rpm} \times$ $5 \mathrm{~min}$ at $4^{\circ} \mathrm{C}$. Nuclei fractions were collected and stored at $-80^{\circ} \mathrm{C}$ until use. HEPES, $\mathrm{KCl}, \mathrm{NaCl}$, EDTA, glycerol, DTT, PMSF, aprotinin, leupeptin, pepstatin A, and IGEPAL were purchased from Sigma-Aldrich.

2.7. Western Blot Analysis. Protein concentrations in the cell, nuclei, and cytoplasmic extracts were measured using the Protein Assay Kit 2 according to the manufacturer's instructions (Bio-Rad Laboratories, Hercules, CA, USA). Thirty micrograms of proteins from the cell or nuclei fractions were separated by SDS-polyacrylamide gel electrophoresis using $10 \%$ polyacrylamide gel. Proteins were then transferred onto polyvinylidene difluoride membranes (Immobilon-P, Millipore, MA, USA). The membranes were blocked overnight using $5 \%$ nonfat milk in $50 \mathrm{mM}$ Tris $/ 150 \mathrm{mM} \mathrm{HCl}(\mathrm{pH}$ 7.5) containing $0.1 \%$ Tween 20 (TBS/Tween). After three 5 -min rinses with TBS/Tween, membranes were probed with polyclonal anti-PPAR $\gamma$, anti-COX-2, anti-p65 NF- $\kappa \mathrm{B}$, or anti-I $\kappa \mathrm{B} \alpha$ (Santa Cruz Biotechnology, Heidelberg, Germany) for $1 \mathrm{~h}$ at room temperature. After three 5 -min rinses with TBS/Tween, horseradish peroxidase- (HRP-) conjugated secondary antibodies goat anti-rabbit or goat anti-mouse IgG (Santa Cruz Biotechnology) were applied for $1 \mathrm{~h}$ at room temperature. Protein bands were visualized using a chemiluminescence detection system (Immun-Star HRP; Bio-Rad Laboratories, Hercules, CA, USA). To normalize protein signals, stripped PVDF membranes were reprobed with monoclonal anti- $\beta$-actin (Sigma-Aldrich) for cytosolic and cell fractions or with polyclonal anti-lamin A/C (Santa Cruz Biotechnology).

2.8. Statistical Analysis. Data were expressed as mean \pm standard deviation. Multiple comparisons were carried out using one-way analysis of variance (ANOVA), followed by Bonferroni post hoc test. SPSS statistical Software, version 14 (SPSS Inc, Chicago, IL, USA), was used for analyses. Significance was defined as $P<0.05$.

\section{Results and Discussion}

3.1. BALF Cytokine Pattern in ARDS Patients. The BALF was collected from twelve adult ARDS patients (9 males and 3 females; age $56 \pm 4$ yrs; pneumonia $n=7$ and sepsis $n=$ 5; Simplified Acute Physiology Score (SAPS) II $42 \pm 18$ ). Proinflammatory cytokines IL- $1 \beta$ and TNF- $\alpha$ were significantly higher in primary pneumonia patients (pulmonary ARDS, ARDSp) than in patients with ARDS originating from sepsis (extrapulmonary ARDS, ARDSexp) (Figure 1(a)). This was also observed for IL- 6 and, in particular, IL-8 content (Figure 1(b)).

It has been extensively documented that in BALF of ARDS patients the content of proinflammatory cytokines is markedly elevated [12]. TNF- $\alpha$ and IL- $1 \beta$ are the main proinflammatory cytokines and they are important in driving the initial lung inflammatory response principally through the stimulation of other cytokines (i.e., IL-6 and IL-8). IL-6 
occupies a critical place in modulating ARDS inflammatory response, while chemokine IL-8 is the major neutrophil chemotactic factor into the alveolar space and it is an early marker for the development of ARDS [10]. In particular, a high content of IL-6 and IL- 8 is correlated with the progression of lung injury as well as with a poor outcome $[8,9]$.

There are many etiologic risk factors for ARDS, which are generally divided into those associated with direct injury (ARDSp) to the lung and those that cause indirect lung injury (ARDSexp) in the setting of a systemic process [17, 39, 40]. Indeed, experimental and clinical studies showed little overall differences in the inflammatory responses between direct and indirect lung injury categories. Thus, identification of the risk factor leading to ARDS in the single patient, regardless of its direct or indirect nature, is rather useful to guide treatment for the underlying disease causing ARDS [1]. Moreover, clinical data supported the theory that an overaggressive and persistent patient inflammatory response, rather than the condition causing lung injury, is the most important factor affecting survival in ARDS patients [41].

\subsection{BALF of ARDS Patients Stimulates A549 Inflammatory} Response. In a previous study, we demonstrated that human alveolar cells (A549) release proinflammatory cytokines (TNF- $\alpha$, IL-6, and IL- 8 ) in the culture medium when challenged with a proinflammatory stimulus such as LPS, suggesting that the alveolar epithelium has a role in the hyperinflammatory response associated with ARDS [32]. Since LPS is an artificial stimulus, in this study we challenged A549 with BALF of ARDS patients. In this study, BALF stimulation induced a proinflammatory response from A549 cells as demonstrated by release of inflammatory cytokines: TNF- $\alpha$ (Figure 2(a)), IL-6 (Figure 2(b)), and IL-8 (Figure 2(c)) while BALF did not elicit anti-inflammatory IL10 release (Figure $2(\mathrm{~d})$ ). These results demonstrated that the pattern of cytokine release of A549 cells exposed to BALF is similar to that elicited by LPS in the previous study.

3.3. Opposite Effects of DHA/AA Ratios on the Cytokine Release from A549 Stimulated Cells. The nutrition support of ARDS patients includes lipids, usually soybean or safflower oilbased emulsions. These emulsions contain more than $50 \%$ of linoleic acid ( $\omega-6)$, a precursor of AA [42], while are deficient, less than $10 \%$, of $\omega-3$ PUFAs (mainly, $\alpha$-linolenic acid). Even though the beneficial effects of $\omega$-3 PUFA have been extensively proven by plenty of experimental preclinical data, conflicting results have been obtained from both clinical trials and human intervention studies [15], in ARDS in particular [28]. Therefore, the debate in the scientific community is still open and a definitive accepted recommendation concerning the use of $\omega-3$ fatty acids in ARDS is still lacking [42]. The observation that optimal $\omega-3$ administration was not only dose-related but was also independently affected by the $\omega-3 / \omega-6$ PUFA ratio has led many authors to focus the attention on the $\omega-3 / \omega-6$ PUFA ratio in nutrition support to modulate inflammation responses. Different $\omega-3 / \omega-6$ PUFA ratios, from $1: 1$ to $1: 4$, have been proposed, but the question of the most favorable $\omega-3 / \omega-6$ PUFA ratio in ARDS patients is not definitely established [43].

In a previous study, we demonstrated that, by affecting $\omega-3 / \omega-6$ ratio in phospholipids cell membranes with $1: 1$ and $1: 2 \mathrm{DHA} / \mathrm{AA}(\omega-3 / \omega-6)$ ratio supply, the balance between proinflammatory and anti-inflammatory cytokines was modulated, thus limiting the A549 LPS-induced hyperinflammatory response. Moreover, we found that ratios with a $\omega$-6 prevalence (i.e., 1:4 and 1:7 DHA/AA) potentiated the effect of LPS stimulus [32]. In the present study, we investigated whether 1:2 DHA/AA ratio was similarly effective in reducing A549 proinflammatory response induced by an ex vivo inflammatory stimulus such as BALF collected from ARDS patients. The 1:2 ratio was preferred to $1: 1$ ratio because it could combine efficacy and decreased risk of immunosuppressive effects. The results clearly indicate that 1:2 DHA/AA treatment significantly reduced the release of proinflammatory cytokines induced by BALF challenge: TNF- $\alpha$ (Figure 2(a)), IL-6 (Figure 2(b)), and IL-8 (Figure 2(c)). Besides, we found that 1:2 DHA/AA treatment was also associated with an increased release of the IL-10 (Figure 2(d)), a potent anti-inflammatory cytokine, confirming that 1:2 DHA/AA was able to modulate the balance between proinflammatory and anti-inflammatory cytokines. Finally, the 1:7 DHA/AA ratio significantly potentiated the BALF inflammatory effects.

Several studies have identified two main key elements in the pathogenesis of ARDS: the occurrence of an imbalance between proinflammatory and anti-inflammatory cytokines [12] and the persistent elevation of proinflammatory mediators [8]. These conditions lead to additional nonpulmonary organ dysfunction which contributes to excess mortality rates in intensive care units [33, 42]. Therefore, strategies for limiting the intensity of lung inflammatory response are of major importance for prognosis and therapy. However, although some pharmacological strategies have proven to be successful in animal studies, human translation of these results has not been so effective on outcome [44].

3.4. Biochemical and Molecular Mechanisms Involved in AntiInflammatory Effects of $\omega-3$ in BALF-Stimulated A549 Cells. Over the last 25 years, the pathophysiology and pharmacology of $\omega$-3 PUFA have been continuously under scrutiny. These FA are able to partly inhibit a number of aspects of inflammation including eicosanoid and cytokine production and bioavailability. The PUFA capacity to modulate different signaling pathways involved in inflammation response has been extensively studied in both physiological and pathological conditions $[14,16]$. The main mechanisms explaining the PUFA role in inflammation and investigated in this study were graphically represented in Figure 3: effects on phospholipid composition of A549 cell membranes, modulation of eicosanoid and cytokine biosynthesis and release, and effects on inflammatory signaling transcription pathways.

3.4.1. Eicosanoid Synthesis. The modulation of eicosanoid production is driven by modification of the FA composition of the phospholipids within cell membranes. PUFAs are rapidly incorporated into cell membrane phospholipids; 


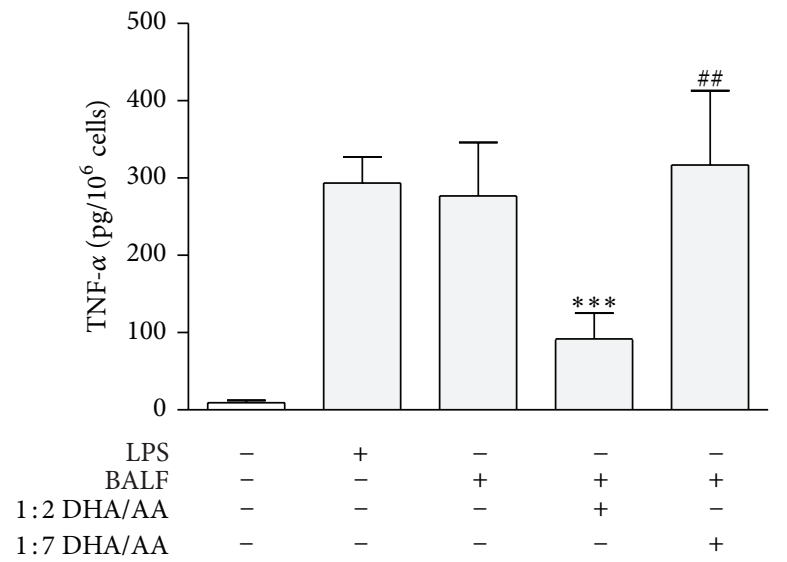

(a)

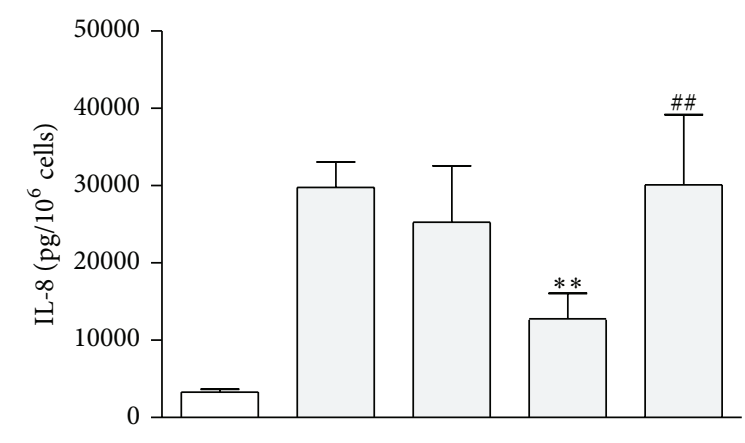

$\begin{array}{rlllll}\text { LPS } & - & + & - & - & - \\ \text { BALF } & - & - & + & + & + \\ 1: 2 \text { DHA/AA } & - & - & - & + & - \\ 1: 7 \text { DHA/AA } & - & - & - & - & +\end{array}$

(c)

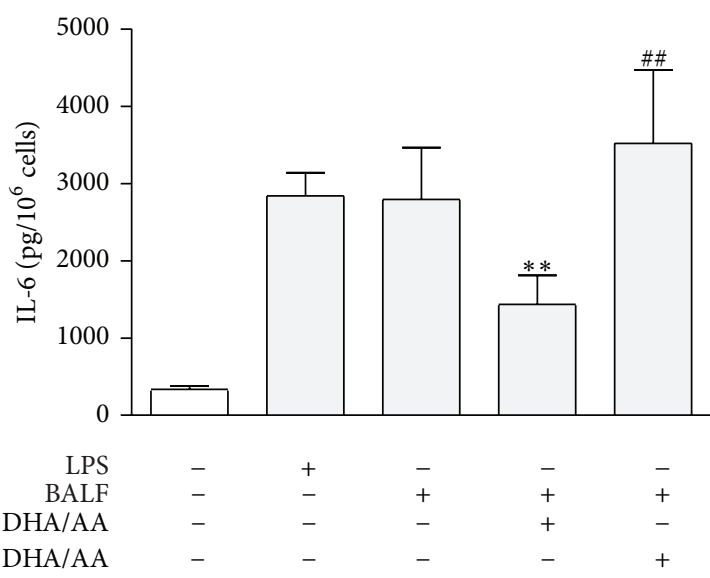

(b)

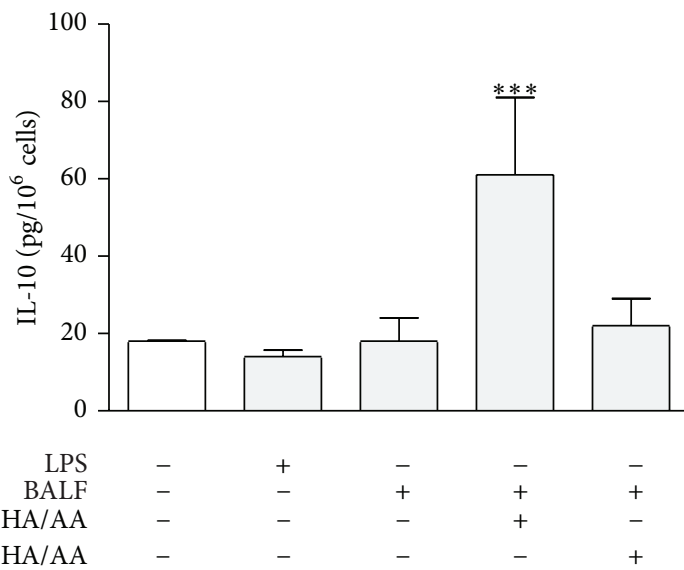

(d)

FIGURE 2: Effects of $\omega-3 / \omega-6$ PUFA ratios on BALF induced cytokine release from A549 cells. (a) TNF- $\alpha$ proinflammatory cytokine release from A549 cells, stimulated with BALF and treated with 1:2 and 1:7 DHA/AA PUFA ratios. (b) IL-6 proinflammatory cytokine release from A549 cells stimulated with BALF and treated with 1:2 and 1:7 DHA/AA ( $\omega-3 / \omega-6)$ PUFA ratios. (c) IL-8 proinflammatory cytokine release from A549 cells stimulated with BALF and treated with 1:2 and 1:7 DHA/AA PUFA ratios. (d) IL-10 anti-inflammatory cytokine release from A549 cells stimulated with BALF and treated with 1:2 and 1:7 DHA/AA PUFA ratios. In each panel, data are presented as picograms (pg) of the indicated cytokine per million cells. Data are presented as mean \pm standard deviation of 12 independent determinations $(n=12)$. PUFA, polyunsaturated fatty acid; BALF, bronchoalveolar lavage fluid; TNF, tumor necrosis factor; DHA, docosahexaenoic acid; AA, arachidonic acid; IL, interleukin; LPS, lipopolysaccharide. ${ }^{* * *} P<0.0011: 2$ DHA/AA versus all. ${ }^{* *} P<0.011: 2$ DHA/AA versus all. ${ }^{\# \#} P<0.011: 7$ DHA/AA versus LPS and BALF.

moreover, the esterification of $\omega-3$ FAs is mainly at the expense of $\omega-6$ AA [14]. Membrane phospholipids PUFAs are precursors of eicosanoids, which are the biologically active lipid mediators of inflammation playing wide ranging roles in inflammation and in regulation of immune function [14]. Eicosanoids originated from AA $\omega-6$ PUFA (2-series prostaglandins (PGs) and 4-series leukotrienes (LTs)) have proinflammatory properties while eicosanoids formed from EPA and DHA $\omega-3$ PUFA (3-series PGs and 5-series LTs) are less active and potentially anti-inflammatory [14]. The balance between proinflammatory and anti-inflammatory eicosanoid synthesis determines the extent of inflammatory reaction [19].

In our previous study, we demonstrated that at the baseline the $\omega-3 / \omega-6$ PUFA ratio in membrane phospholipids of A549 was 1:5 and that it can be changed by challenging the cells with $1: 1$ or 1:2 DHA/AA ratios [32]. Here, we examined the DHA and AA content in membrane phospholipids of A549 BALF-stimulated and challenged with $1: 2$ or $1: 7$ ratios DHA/AA (Figure 4). At baseline in A549 membrane phospholipids the $\omega-6$ PUFA fraction is predominant, with a $\omega-3 / \omega-6$ ratio of $1: 5$. Notably, cell treatment with both DHA/AA ratios reduced the AA content in A549 membrane phospholipids, but the 1:2 DHA/AA ratio also significantly increased the DHA percentage content. The supply of $1: 2$ DHA/AA ratio reversed the baseline predominance of $\omega-6$ over $\omega-3$ in the $\omega-3 / \omega-6$ PUFA ratio in membrane phospholipids of A549 cells.

Eicosanoid biosynthesis begun when an inflammatory stimulus activates phospholipases, which are the enzymes that cleave the fatty acid precursors from the membrane phospholipids. Released PUFAs are then converted into 


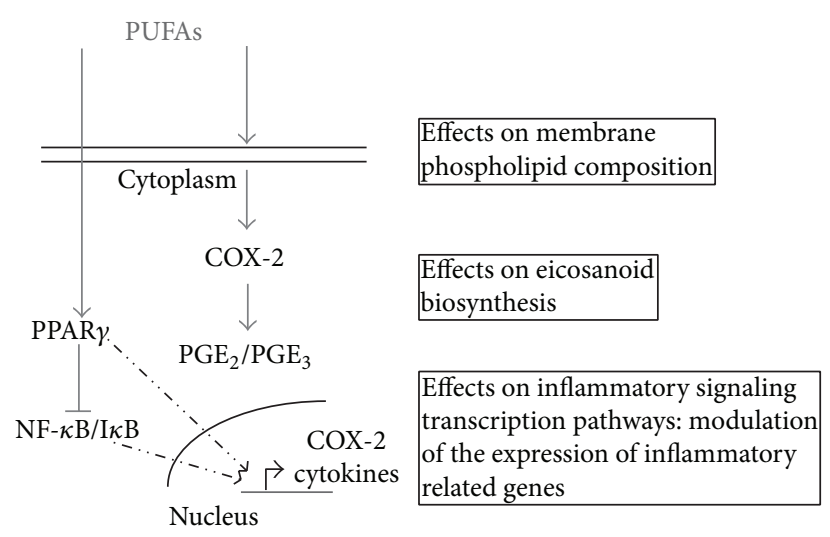

FigURE 3: Schematic representation of PUFA mechanism of action in BALF-stimulated A549 cells. PUFAs, polyunsaturated fatty acids; COX, cycloxygenase; PG, prostaglandin; PPAR, peroxisome proliferator-activated receptor; NF- $\kappa \mathrm{B}$, nuclear factor-kappa $\mathrm{B} ; \mathrm{I} \kappa \mathrm{B}$, inhibitor of NF- $\kappa$ B.

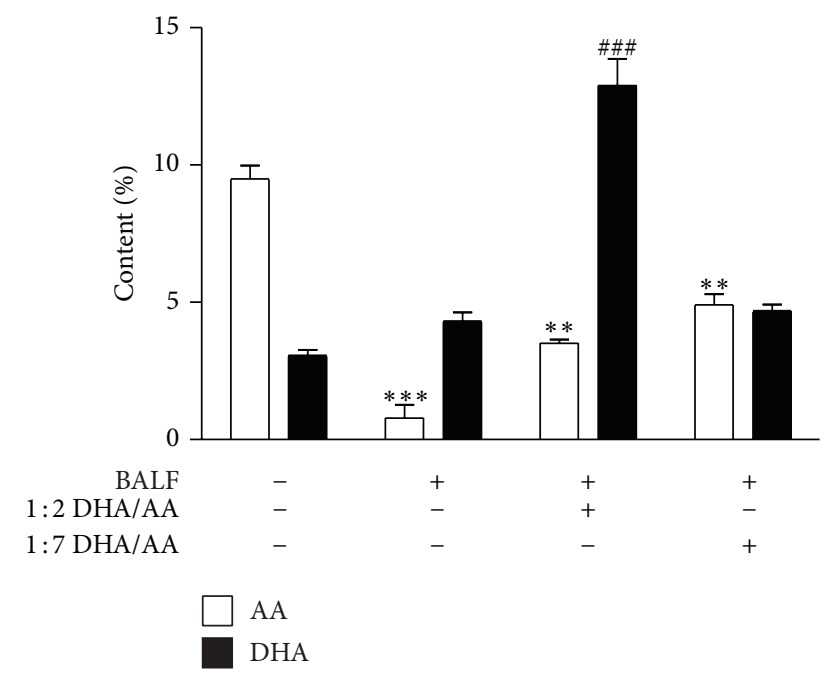

FIgURE 4: Effects of $\omega-3 / \omega-6$ PUFA ratios on the percentage content of AA and DHA in A549 membrane phospholipids. Relative percentage content of AA (white bars) and DHA (black bars) in phospholipids of A549 cell membranes stimulated with BALF and treated with $50 \mu \mathrm{M} 1: 2$ or $1: 7$ DHA/AA PUFA ratios. Data are presented as percentage content of AA and DHA in the membrane phospholipids of unstimulated or stimulated A549 cells as indicated. Data are presented as mean \pm standard deviation of 4 independent experiments $(n=4)$. PUFA, polyunsaturated fatty acid; AA, arachidonic acid; DHA, docosahexaenoic acid; BALF, bronchoalveolar lavage fluid. ${ }^{* * *} P<0.001$ BALF versus unstimulated. ${ }^{* *} P<0.01$ $1: 2$ and $1: 7$ DHA/AA versus all. ${ }^{\# \#} P<0.001$ versus all.

eicosanoids, mainly PGs and tromboxanes by cycloxygenases (COXs) and LTs by lipoxygenases (LOXs) [14].

There are two COX isoforms: the COX-1 that is constitutively expressed in almost all cells and the COX-2 that is induced in many cell types by a broad range of proinflammatory agents [45]. The COX-2, also known as PGendoperoxide synthase, is the key enzyme in PG synthesis from AA.
To verify whether in our experimental model there was a modulation of PG biosynthesis, we analyzed the content of COX-2. The results indicated that in response to BALF stimulus A549 cells increased by 2.5-fold the expression of COX-2 (Figure 5(a)) and produce a significant amount of $\mathrm{PGE}_{2}$ (Figure 5(b)). Noteworthy, even if COX-2 is the inducible form of COX, A549 cells express the enzyme constitutively [46]. According to Yang et al. [46], we found that this level of COX-2 expression was not associated with a PG synthesis (Figure 5(b)) in unstimulated A549.

The 1:2 DHA/AA treatment significantly restored the expression of the enzyme at the level of unstimulated cells (Figure 5(a)). This effect was significantly associated with a reduction of $\mathrm{PGE}_{2}$ release and an induction of $\mathrm{PGE}_{3}$ synthesis (Figure 5(b)), suggesting that the enzyme was in an active form. The 1:7 DHA/AA ratio induced a less significant inhibitory effect on the COX-2 content and the $\mathrm{PGE}_{2}$ synthesis. The differences in COX- 2 content could be correlated with the $\mathrm{PGE}_{2}$-dependent amplification of the enzyme [45]. These results indicate that both $\omega-3$ and $\omega-6$ PUFAs reduce the expression of COX-2 induced by inflammatory stimuli such as BALF but with a different extent. These effects on $\mathrm{PGE}_{2}$ content well correlated with the availability of AA substrate into membrane phospholipids (Figure 4). Moreover, treatment with 1:2 DHA/AA also induced a significant increase in $\mathrm{PGE}_{3}$ content, a less potent inflammatory PG. This finding well correlated with the increased DHA content in A549 membrane phospholipids (Figure 4). Notably, $\mathrm{PGE}_{3}$ is a less potent inducer of COX-2 gene expression in fibroblasts and of IL-6 production by macrophages compared with $\mathrm{PGE}_{2}$ [13]. In accordance with Yang et al's data [46], our results confirm that exposure of alveolar cells to $\omega-3$ PUFA determines a decrease in the COX-2-mediated formation of $\mathrm{PGE}_{2}$ and an increase in the level of $\mathrm{PGE}_{3}$.

3.4.2. Inflammatory Signaling Transcription Pathways: NF- $\kappa B$ and PPAR $\gamma$. The nuclear factor (NF- $\kappa \mathrm{B})$ is a key transcription factor involved in upregulation of inflammatory cytokines, adhesion molecules, and COX-2 genes [47]. Activation of NF$\kappa \mathrm{B}$ transcription factor has been implicated in a number of inflammation-related pathologies [14]. The p65 and p50 NF$\kappa \mathrm{B}$ heterodimers are maintained inactive in the cytosol by the binding with an inhibitory protein, namely, inhibitor of NF$\kappa \mathrm{B}(\mathrm{I} \kappa \mathrm{B})$ [48]. Proinflammatory stimuli induce phosphorylation, ubiquitination, and proteasome mediated degradation of $\mathrm{I} \kappa \mathrm{B}$, allowing NF- $\kappa \mathrm{B}$ translocation into the nucleus and NF- $\kappa \mathrm{B}$ target gene transcription [48]. The inhibitory role of $\omega-3$ PUFAs on NF- $\kappa$ B pathway has been demonstrated in several experimental models and pathological conditions [19]. Different authors demonstrated that $\omega-3$ decreased TNF- $\alpha$ expression through the prevention of NF- $\kappa \mathrm{B}$ activation by inhibiting $\mathrm{I} \kappa \mathrm{B}$ phosphorylation and consequently preventing $\mathrm{NF}-\kappa \mathrm{B}$ translocation into the nucleus [14]. The correlation between NF- $\kappa$ B activation and cytokine content in BALF of ARDS patients was previously demonstrated by Nys et al. [49]. In order to understand the role of 1:2 DHA/AA treatment on the NF- $\kappa \mathrm{B}$ pathway, we analyzed the expression of p 65 NF- $\kappa$ B both in the cytoplasmic and the nuclear fraction of A549 BALF-stimulated cells (Figures 6(a) and 6(b)). 

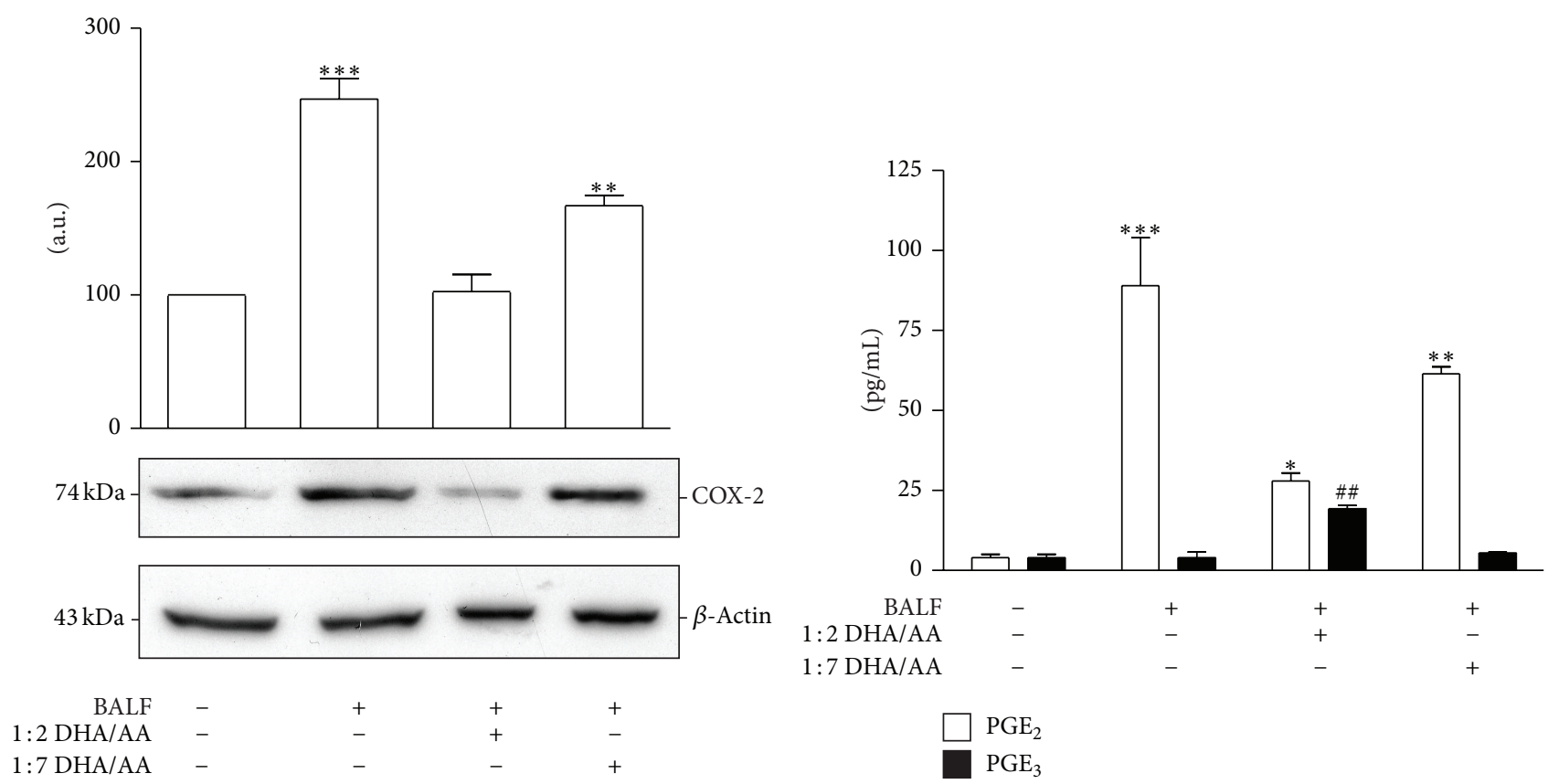

(a)

(b)

FIgURE 5: Effects of $\omega-3 / \omega-6$ PUFA ratios on $\mathrm{PGE}_{2}$ and $\mathrm{PGE}_{3}$ synthesis and release. (a) COX-2 relative protein content in A549 cells, stimulated with BALF and treated with $50 \mu \mathrm{M} 1: 2$ and 1:7 DHA/AA PUFA ratios. Data are expressed as "a.u." (arbitrary units) of the densitometric values, normalized on the corresponding $\beta$-actin. The value of unstimulated cells was arbitrarily set as 100 . Data are presented as mean \pm standard deviation of 6 independent determinations $(n=6)$. The image is representative of all the WB experiments. (b) PGE 2 (white bars) and $\mathrm{PGE}_{3}$ (black bars) content in culture media of A549 cells, stimulated with BALFs and treated with 50 $\mu \mathrm{M}$ 1:2 or 1:7 DHA/AA ratio. Data are presented as picograms (pg) of the indicated PG per mL. Data are presented as mean \pm standard deviation of 12 independent determinations $(n=12)$. PUFA, polyunsaturated fatty acid; PG, prostaglandin; COX, cycloxygenase; BALF, bronchoalveolar lavage fluid; DHA, docosahexaenoic acid; AA, arachidonic acid; WB, western blot. ${ }^{* * *} P<0.001$ BALF versus unstimulated cells and $1: 2$ DHA/AA. ${ }^{* *} P<0.011: 7$ DHA/AA versus all. ${ }^{*} P<0.051: 2$ DHA/AA versus unstimulated cells. ${ }^{\# \#} P<0.011: 2$ DHA/AA versus all.

The results demonstrated that 1:2 DHA/AA treatment decreased NF- $\kappa \mathrm{B}$ content, in the nuclear fraction in particular, indicating an inhibition of its activation. The results were confirmed by the finding that $1: 2 \mathrm{DHA} / \mathrm{AA}$ treatment increased the content of $\mathrm{I} \kappa \mathrm{B} \alpha$, one of the isoforms of $\mathrm{I} \kappa \mathrm{B}$ (Figure $6(\mathrm{c})$ ). These results suggested that the antiinflammatory effects of $1: 2 \mathrm{DHA} / \mathrm{AA}$ treatment were associated with the inhibition of the NF- $\kappa$ B inflammatory pathway. Since, genes of IL- $1 \beta$, IL- 6 , and TNF- $\alpha$, as well as COX-2, are regulated by NF- $\kappa \mathrm{B}[50]$, we can speculate that the reduction of proinflammatory cytokine release and the inhibition of COX-2 expression were mediated by the inhibition of NF- $\kappa \mathrm{B}$ transcriptional activity.

Peroxisome proliferator-activated receptors (PPARs) are ligand-activated nuclear transcription factors encoded by different genes. PPARs include 3 subtypes $(\alpha, \beta$, and $\gamma)$, which are characterized by unique functions such as ligand specificities and tissue distribution [51]. PPAR ligands encompass endogenous metabolites such as prostanoids and PUFAs, as well as synthetic drugs such as fibrates and thiazolidinediones. In macrophages, activation of PPAR $\gamma$ negatively influences the production of inflammatory cytokines like TNF- $\alpha$, IL-6, and IL- $1 \beta$ [52]. It has been demonstrated that most of the effects of PPARs on cytokine expression result from crosstalk with other transcriptional factors and in particular with NF- $\kappa \mathrm{B}$ [53]. To verify if also in our experimental model the anti-inflammatory effects of $\omega$-3 were correlated with PPAR activation, we determined the PPAR $\gamma$ content (Figure 7). The results indicated that the proinflammatory stimulus was associated with the inhibition of PPAR $\gamma$ expression. Moreover, as we previously demonstrated [35], in the present study we found both PUFA ratios were associated with an increased PPAR $\gamma$ content but to a greater extent with the $1: 2$ DHA/AA treatment.

Summarizing, we speculate that in our experimental model the anti-inflammatory effects of $1: 2 \mathrm{DHA} / \mathrm{AA}$ treatment could be mediated by reduction of COX-2 expression, decrease of NF- $\kappa \mathrm{B}$ translocation into the nucleus, and $\operatorname{PPAR} \gamma$ activation. Thus, the results presented herein give further insight into the mechanisms involved in the antiinflammatory effect of $\omega$-3 PUFAs.

3.4.3. Resolvins and Protectins. Recent studies have identified n-3 PUFAs as precursors of a distinct set of lipid mediators that probably act through distinct receptors to exert their anti-inflammatory effects. These new n-3 PUFA-derived, anti-inflammatory mediators have been named resolvins and protectins. For an understanding of resolvin and protectin 

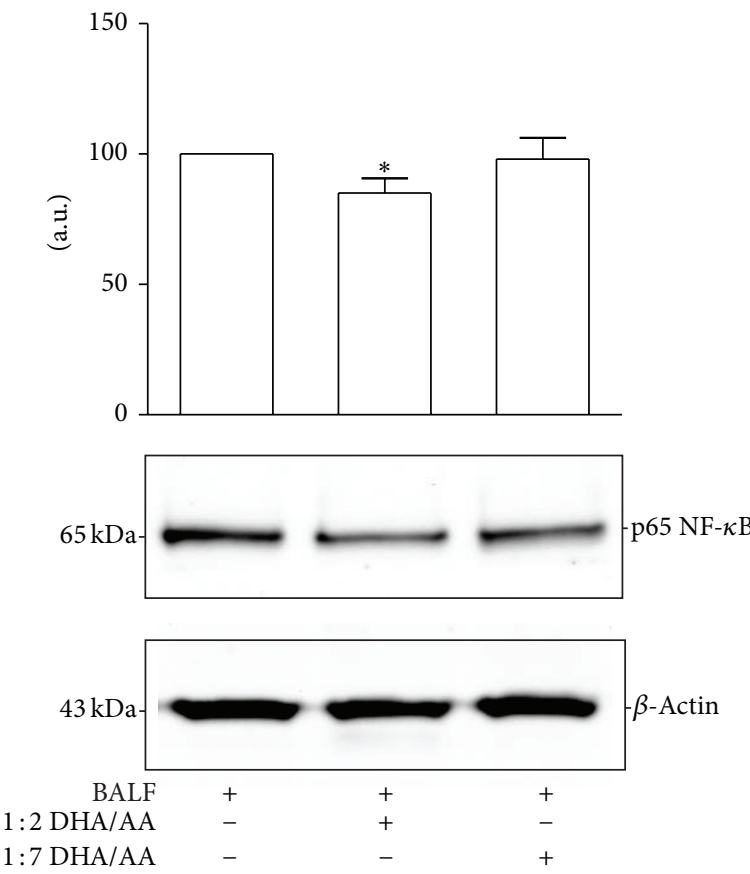

(a)
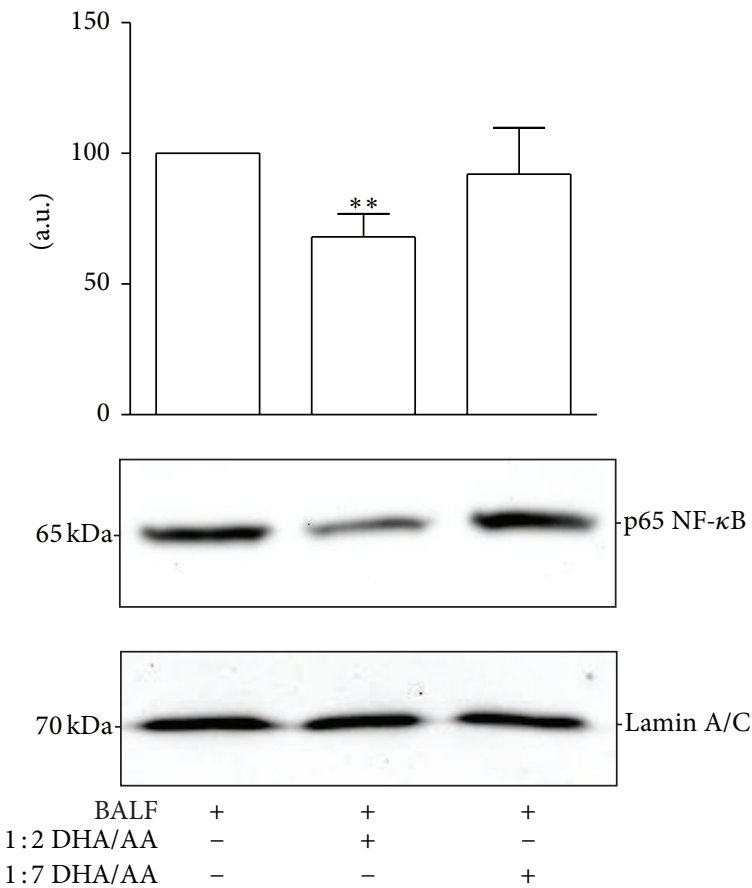

(b)
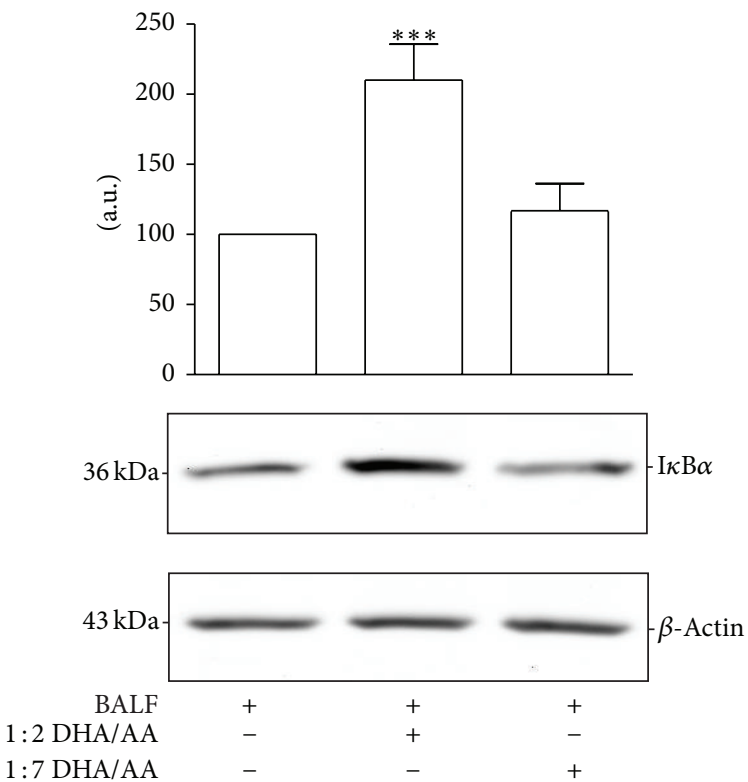

(c)

Figure 6: Effects of $\omega-3 / \omega-6$ PUFA ratios on NF- $\kappa$ B. (a) p65 NF- $\kappa$ B relative protein content in the cytoplasmic fraction of A549 cells, stimulated with BALF and treated with $50 \mu \mathrm{M} \mathrm{1:2} \mathrm{or} \mathrm{1:7} \mathrm{DHA/AA} \mathrm{ratios.} \mathrm{Data} \mathrm{are} \mathrm{expressed} \mathrm{as} \mathrm{"a.u."} \mathrm{(arbitrary} \mathrm{units)} \mathrm{of} \mathrm{the} \mathrm{densitometric}$ values, normalized on the corresponding $\beta$-actin. The value of BALF was arbitrarily set as 100. Data are presented as mean \pm standard deviation of 6 independent determinations $(n=6)$. The image is representative of all the WB experiments. (b) NF- $\kappa$ B relative protein content in the nuclear fraction of A549 cells, stimulated with BALF and treated with 50 $\mu \mathrm{M} 1: 2$ or 1:7 DHA/AA ratios. Data are expressed as "a.u." (arbitrary units) of the densitometric values, normalized on the corresponding lamin A/C. The value of BALF was arbitrarily set as 100. Data are presented as mean \pm standard deviation of 6 independent determinations $(n=6)$. (c) I $\kappa \mathrm{B} \alpha$ relative protein content in the cytoplasmic fraction of A549 cells, stimulated with BALF and treated with $50 \mu \mathrm{M} \mathrm{1:2} \mathrm{or} \mathrm{1:7} \mathrm{DHA/AA} \mathrm{ratios.} \mathrm{Data} \mathrm{are} \mathrm{expressed} \mathrm{as} \mathrm{"a.u."} \mathrm{(arbitrary}$ units) of the densitometric values, normalized on the corresponding $\beta$-actin. The value of BALF was arbitrarily set as 100 . Data are presented as mean \pm standard deviation of 6 independent determinations $(n=6)$. The image is representative of all the WB experiments. PUFA, polyunsaturated fatty acid; NF- $\kappa$ B, nuclear factor-kappa B; BALF, bronchoalveolar lavage fluid; DHA, docosahexaenoic acid; AA, arachidonic acid; WB, western blot; I $\kappa$ B, inhibitor of NF- $\kappa B$ B ${ }^{*} P<0.051: 2$ DHA/AA versus all. ${ }^{* *} P<0.011: 2$ DHA/AA versus all. ${ }^{* * *} P<0.0011: 2$ DHA/AA versus all. 

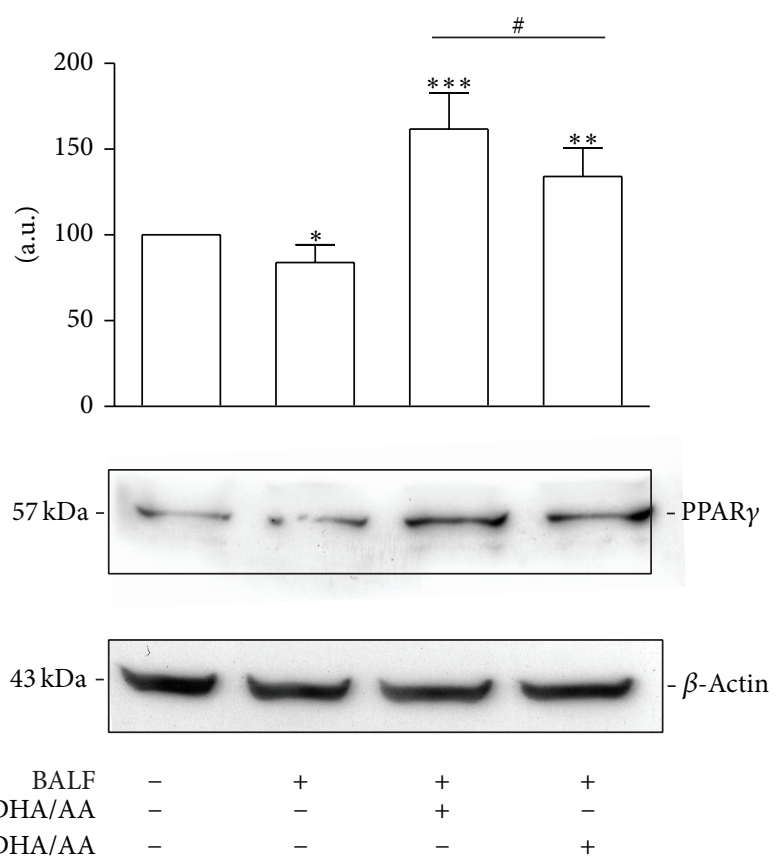

Figure 7: Effects of $\omega-3 / \omega-6$ PUFA ratios on PPAR $\gamma$ expression. PPAR $\gamma$ relative protein content in A549 cells stimulated with BALF and treated with $50 \mu \mathrm{M} 1: 2$ or 1:7 DHA/AA ratios. Data are expressed as "a.u." (arbitrary units) of the densitometric values, normalized on the corresponding $\beta$-actin. The value of unstimulated cells was arbitrarily set as 100 . Data are presented as mean \pm standard deviation of 6 independent determinations $(n=6)$. The image is representative of all the WB experiments. PUFA, polyunsaturated fatty acid; PPAR, peroxisome proliferator-activated receptor; BALF, bronchoalveolar lavage fluid; DHA, docosahexaenoic acid; AA, arachidonic acid; WB, western blot. ${ }^{*} P<0.05$ BALF versus unstimulated cells. ${ }^{* *} P<0.011: 7$ DHA/AA versus BALF and unstimulated cells. ${ }^{* * *} P<0.001$ 1:2 DHA/AA versus BALF and

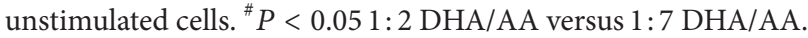

formation see Weylandt et al. [54] for a complete review. Briefly, these lipid mediators might offer an important new concept to explain the protective effect of n-3 PUFAs in a wide variety of disease models. In particular, DHA constitutes the origin for the D-series resolvins-mainly, resolvin D1 (RvD1) - as well as (neuro-) protectin D1.

In this study, we did not investigate resolvins or protectins in our experimental model; however, previous experimental studies showed that RvD1 had potent anti-inflammatory effects in several disease models including lung injury. RvD1 could modulate the balance between proinflamatory and anti-inflammatory cytokines, alter the response of the host to pulmonary bacterial infection, and affect the early outcome of infection [55]. Wang et al. showed that RvD1 improved survival rate and attenuated ALI in mice induced by LPS; specifically, RvD1 inhibited increases in TNF- $\alpha$ and IL-6 production in the BALF, reduced expression of COX-2, and inhibited activation of NF- $\kappa \mathrm{B}[56]$.

\section{Conclusions}

ARDS is an inflammatory disease whose clinical severity mostly depends on the grade of inflammation. Cytokines and eicosanoids are key elements in the pathogenesis and outcome of ARDS.

To the best of our knowledge, this is the first study reporting the role of $\omega$-3 to $\omega$-6 PUFA ratio in the modulation of release of four cytokines (TNF- $\alpha$, IL- 6 , IL-8, and IL10) and two prostaglandins $\left(\mathrm{PGE}_{2}\right.$ and $\left.\mathrm{PGE}_{3}\right)$ in human alveolar cells exposed to BALF of ARDS patients. Differently from our earlier study, the present study has some original features. First, we challenged alveolar cells with an ex vivo inflammatory stimulus and not with LPS. Second, the number of experiments was greater (12 versus 4 ). Third, we also investigated the PG release as well as the content of COX2. Finally, we examined the more important inflammatory signal transduction pathways (NF- $\kappa \mathrm{B}$ and PPAR $\gamma$ ) at work in this experimental inflammatory cell model.

The results of this study demonstrated that shifting the PUFA supply from $\omega-6$ to $\omega-3$ decreased the release of proinflammatory cytokines and $\mathrm{PGE}_{2}$ in human alveolar cells challenged with BALF of ARDS patients. Moreover, these data confirmed our previous finding that a predominance of AA in PUFA supply determined a more aggressive proinflammatory response. Finally, these data provide a contribution to support the biochemical basis for current recommendations [57-60] to shift the lipid supply from $\omega-6$ to $\omega-3$ PUFA in the nutrition support of ARDS patients.

In conclusion, there are good experimental evidence and convincing rationale according to the $\omega$-3 PUFA use in ARDS patients [61]; however, questions still remain to be answered regarding the in vivo effects of these PUFAs.

\section{Conflict of Interests}

The authors declare that there is no conflict of interests regarding the publication of this paper.

\section{Authors' Contribution}

Paolo Cotogni and Antonella Trombetta contributed equally to this work.

\section{Acknowledgments}

This work was partially supported by the Regione Piemonte (Italy) (Grant nos. 19700/27.001, 1837/27.001 to P. Cotogni). The authors thank Antonella Meloni for her critical revision of the paper.

\section{References}

[1] N. D. Ferguson, E. Fan, L. Camporota et al., "The Berlin definition of ARDS: an expanded rationale, justification, and supplementary material," Intensive Care Medicine, vol. 38, no. 10, pp. 1573-1582, 2012. 
[2] ARDS Definition Task Force, V. M. Ranieri, G. D. Rubenfeld et al., "Acute respiratory distress syndrome: the Berlin definition," The Journal of the American Medical Association, vol. 307, no. 23, pp. 2526-2533, 2012.

[3] P. Terragni, C. Faggiano, and V. Ranieri Marco, "Extracorporeal membrane oxygenation in adult patients with acute respiratory distress syndrome," Current Opinion in Critical Care, vol. 20, no. 1, pp. 86-91, 2014.

[4] J. Pugin, G. Verghese, M.-C. Widmer, and M. A. Matthay, "The alveolar space is the site of intense inflammatory and profibrotic reactions in the early phase of acute respiratory distress syndrome," Critical Care Medicine, vol. 27, no. 2, pp. 304-312, 1999.

[5] K. Mayer, S. Gokorsch, C. Fegbeutel et al., "Parenteral nutrition with fish oil modulates cytokine response in patients with sepsis," American Journal of Respiratory and Critical Care Medicine, vol. 167, no. 10, pp. 1321-1328, 2003.

[6] W. L. Lee and G. P. Downey, "Neutrophil activation and acute lung injury," Current Opinion in Critical Care, vol. 7, no. 1, pp. $1-7,2001$.

[7] D. W. Shimabukuro, T. Sawa, and M. A. Gropper, "Injury and repair in lung and airways," Critical Care Medicine, vol. 31, no. 8, supplement, pp. S524-S531, 2003.

[8] G. U. Meduri, S. Headley, G. Kohler et al., "Persistent elevation of inflammatory cytokines predicts a poor outcome in ARDS: plasma IL- $1 \beta$ and IL- 6 levels are consistent and efficient predictors of outcome over time," Chest, vol. 107, no. 4, pp. 1062-1073, 1995.

[9] G. U. Meduri, G. Kohler, S. Headley, E. Tolley, F. Stentz, and A. Postlethwaite, "Inflammatory cytokines in the BAL of patients with ARDS: persistent elevation over time predicts poor outcome," Chest, vol. 108, no. 5, pp. 1303-1314, 1995.

[10] S. C. Donnelly, R. M. Strieter, P. T. Reid et al., "The association between mortality rates and decreased concentrations of interleukin-10 and interleukin-1 receptor antagonist in the lung fluids of patients with the adult respiratory distress syndrome," Annals of Internal Medicine, vol. 125, no. 3, pp. 191-196, 1996.

[11] L. Armstrong and A. B. Millar, "Relative production of tumour necrosis factor and interleukin 10 in adult respiratory distress syndrome," Thorax, vol. 52, no. 5, pp. 442-446, 1997.

[12] W. Y. Park, R. B. Goodman, K. P. Steinberg et al., "Cytokine balance in the lungs of patients with acute respiratory distress syndrome," American Journal of Respiratory and Critical Care Medicine, vol. 164, no. 10, part 1, pp. 1896-1903, 2001.

[13] P. C. Calder, " $n-3$ Polyunsaturated fatty acids, inflammation, and inflammatory diseases," The American Journal of Clinical Nutrition, vol. 83, supplement 6, pp. 1505S-1519S, 2006.

[14] P. C. Calder, " $n-3$ Fatty acids, inflammation and immunity: new mechanisms to explain old actions," Proceedings of the Nutrition Society, vol. 72, no. 3, pp. 326-336, 2013.

[15] P. C. Calder, "Omega-3 polyunsaturated fatty acids and inflammatory processes: nutrition or pharmacology?” British Journal of Clinical Pharmacology, vol. 75, no. 3, pp. 645-662, 2013.

[16] G. Calviello, H. M. Su, K. H. Weylandt, E. Fasano, S. Serini, and A. Cittadini, "Experimental evidence of $\omega$-3 polyunsaturated fatty acid modulation of inflammatory cytokines and bioactive lipid mediators: their potential role in inflammatory, neurodegenerative, and neoplastic diseases," BioMed Research International, vol. 2013, Article ID 743171, 13 pages, 2013.

[17] S. K. Leaver and T. W. Evans, "Acute respiratory distress syndrome," British Medical Journal, vol. 335, no. 7616, pp. 389394, 2007.
[18] K. Mayer and W. Seeger, "Fish oil in critical illness," Current Opinion in Clinical Nutrition \& Metabolic Care, vol. 11, no. 2, pp. 121-127, 2008.

[19] P. Singer, H. Shapiro, M. Theilla, R. Anbar, J. Singer, and J. Cohen, "Anti-inflammatory properties of omega-3 fatty acids in critical illness: novel mechanisms and an integrative perspective," Intensive Care Medicine, vol. 34, no. 9, pp. 1580-1592, 2008.

[20] I. Breil, T. Koch, A. Heller et al., "Alteration of n-3 fatty acid composition in lung tissue after short- term infusion of fish oil emulsion attenuates inflammatory vascular reaction," Critical Care Medicine, vol. 24, no. 11, pp. 1893-1902, 1996.

[21] P. Yang, D. Chan, E. Felix et al., "Formation and antiproliferative effect of prostaglandin $\mathrm{E}_{3}$ from eicosapentaenoic acid in human lung cancer cells," Journal of Lipid Research, vol. 45, no. 6, pp. 1030-1039, 2004.

[22] D. L. Bryan, K. D. Forsyth, P. H. Hart, and R. A. Gibson, "Polyunsaturated fatty acids regulate cytokine and prostaglandin $\mathrm{E}_{2}$ production by respiratory cells in response to mast cell mediators," Lipids, vol. 41, no. 12, pp. 1101-1107, 2006.

[23] J. E. Gadek, S. J. DeMichele, M. D. Karlstad et al., "Effect of enteral feeding with eicosapentaenoic acid, gamma-linolenic acid, and antioxidants in patients with acute respiratory distress syndrome. Enteral Nutrition in ARDS Study Group," Critical Care Medicine, vol. 27, no. 8, pp. 1409-1420, 1999.

[24] P. Singer, M. Theilla, H. Fisher, L. Gibstein, E. Grozovski, and J. Cohen, "Benefit of an enteral diet enriched with eicosapentaenoic acid and gamma-linolenic acid in ventilated patients with acute lung injury," Critical Care Medicine, vol. 34, no. 4, pp. 1033-1038, 2006.

[25] A. Pontes-Arruda, A. M. A. Aragão, and J. D. Albuquerque, "Effects of enteral feeding with eicosapentaenoic acid, $\gamma$ linolenic acid, and antioxidants in mechanically ventilated patients with severe sepsis and septic shock," Critical Care Medicine, vol. 34, no. 9, pp. 2325-2333, 2006.

[26] T. Grau-Carmona, V. Morán-García, A. García-de-Lorenzo et al., "Effect of an enteral diet enriched with eicosapentaenoic acid, gamma-linolenic acid and anti-oxidants on the outcome of mechanically ventilated, critically ill, septic patients," Clinical Nutrition, vol. 30, no. 5, pp. 578-584, 2011.

[27] R. D. Stapleton, T. R. Martin, N. S. Weiss et al., "A phase II randomized placebo-controlled trial of omega-3 fatty acids for the treatment of acute lung injury," Critical Care Medicine, vol. 39, no. 7, pp. 1655-1662, 2011.

[28] T. W. Rice, A. P. Wheeler, B. T. Thompson, B. P. DeBoisblanc, J. Steingrub, and P. Rock, "Enteral omega-3 fatty acid, $\gamma$-linolenic acid, and antioxidant supplementation in acute lung injury," The Journal of the American Medical Association, vol. 306, no. 14, pp. 1574-1581, 2011.

[29] S. Friesecke, C. Lotze, J. Köhler, A. Heinrich, S. B. Felix, and P. Abel, "Fish oil supplementation in the parenteral nutrition of critically ill medical patients: a randomised controlled trial," Intensive Care Medicine, vol. 34, no. 8, pp. 1411-1420, 2008.

[30] V. M. Barbosa, E. A. Miles, C. Calhau, E. Lafuente, and P. C. Calder, "Effects of a fish oil containing lipid emulsion on plasma phospholipid fatty acids, inflammatory markers, and clinical outcomes in septic patients: a randomized, controlled clinical trial," Critical Care, vol. 14, no. 1, article R5, 11 pages, 2010.

[31] A. Gupta, D. Govil, S. Bhatnagar et al., "Efficacy and safety of parenteral omega 3 fatty acids in ventilated patients with acute lung injury," Indian Journal of Critical Care Medicine, vol. 15, no. 2, pp. 108-113, 2011. 
[32] P. Cotogni, G. Muzio, A. Trombetta, V. M. Ranieri, and R. A. Canuto, "Impact of the $\omega-3$ to $\omega-6$ polyunsaturated fatty acid ratio on cytokine release in human alveolar cells," Journal of Parenteral and Enteral Nutrition, vol. 35, no. 1, pp. 114-121, 2011.

[33] V. M. Ranieri, P. M. Suter, C. Tortorella et al., "Effect of mechanical ventilation on inflammatory mediators in patients with acute respiratory distress syndrome: a randomized controlled trial," Journal of the American Medical Association, vol. 282, no. 1, pp. 54-61, 1999.

[34] P. P. Terragni, G. Rosboch, A. Tealdi et al., "Tidal hyperinflation during low tidal volume ventilation in acute respiratory distress syndrome," American Journal of Respiratory and Critical Care Medicine, vol. 175, no. 2, pp. 160-166, 2007.

[35] G. Muzio, A. Trombetta, M. Maggiora et al., "Arachidonic acid suppresses growth of human lung tumor A549 cells through down-regulation of ALDH3A1 expression," Free Radical Biology and Medicine, vol. 40, no. 11, pp. 1929-1938, 2006.

[36] A. Trombetta, M. Maggiora, G. Martinasso, P. Cotogni, R. A. Canuto, and G. Muzio, "Arachidonic and docosahexaenoic acids reduce the growth of A549 human lung-tumor cells increasing lipid peroxidation and PPARs," Chemico-Biological Interactions, vol. 165, no. 3, pp. 239-250, 2007.

[37] J. Folch, M. Lees, and G. H. Sloane Stanley, "A simple method for the isolation and purification of total lipides from animal tissues," The Journal of Biological Chemistry, vol. 226, no. 1, pp. 497-509, 1957.

[38] L. D. Metcalfe, J. R. Pelka, and A. A. Schmitz, "Rapid preparation of fatty acid esters from lipids for gas chromatographic analysis," Analytical Chemistry, vol. 38, no. 3, pp. 514-515, 1966.

[39] L. B. Ware and M. A. Matthay, "The acute respiratory distress syndrome," The New England Journal of Medicine, vol. 342, no. 18, pp. 1334-1349, 2000.

[40] P. Pelosi, D. D’Onofrio, D. Chiumello et al., "Pulmonary and extrapulmonary acute respiratory distress syndrome are different," The European Respiratory Journal, Supplement, vol. 22, no. 42, pp. 48s-56s, 2003.

[41] A. S. Headly, E. Tolley, and G. U. Meduri, "Infections and the inflammatory response in acute respiratory distress syndrome," Chest, vol. 111, no. 5, pp. 1306-1321, 1997.

[42] M. Hecker, J. Ott, C. Sondermann et al., "Immunomodulation by fish-oil containing lipid emulsions in murine acute respiratory distress syndrome," Critical Care, vol. 18, no. 2, article R85, 2014.

[43] B. A. Mizock, "Nutritional support in acute lung injury and acute respiratory distress syndrome," Nutrition in Clinical Practice, vol. 16, no. 6, pp. 319-328, 2001.

[44] A. Dushianthan, M. P. W. Grocott, A. D. Postle, and R. Cusack, "Acute respiratory distress syndrome and acute lung injury," Postgraduate Medical Journal, vol. 87, no. 1031, pp. 612-622, 2011.

[45] D. Bagga, L. Wang, R. Farias-Eisner, J. A. Glaspy, and S. T. Reddy, "Differential effects of prostaglandin derived from $\omega-6$ and $\omega-3$ polyunsaturated fatty acids on COX-2 expression and IL-6 secretion," Proceedings of the National Academy of Sciences of the United States of America, vol. 100, no. 4, pp. 1751-1756, 2003.

[46] P. Yang, C. Cartwright, D. Chan et al., "Anticancer activity of fish oils against human lung cancer is associated with changes in formation of PGE2 and PGE3 and alteration of Akt phosphorylation," Molecular Carcinogenesis, vol. 53, no. 7, pp. 566-577, 2013.
[47] A. Kumar, Y. Takada, A. M. Boriek, and B. B. Aggarwal, "Nuclear factor- $\kappa$ B: its role in health and disease," Journal of Molecular Medicine, vol. 82, no. 7, pp. 434-448, 2004.

[48] M. Nys, G. Deby-Dupont, Y. Habraken et al., "Bronchoalveolar lavage fluids of ventilated patients with acute lung injury activate NF-kappaB in alveolar epithelial cell line: role of reactive oxygen/nitrogen species and cytokines," Nitric Oxide, vol. 9, no. 1, pp. 33-43, 2003.

[49] M. Nys, G. Deby-Dupont, Y. Habraken et al., "Bronchoalveolar lavage fluids of patients with lung injury activate the transcription factor nuclear factor- $\kappa \mathrm{B}$ in an alveolar cell line," Clinical Science, vol. 103, no. 6, pp. 577-585, 2002.

[50] A. Mullen, C. E. Loscher, and H. M. Roche, "Anti-inflammatory effects of EPA and DHA are dependent upon time and doseresponse elements associated with LPS stimulation in THP-1derived macrophages," The Journal of Nutritional Biochemistry, vol. 21, no. 5, pp. 444-450, 2010.

[51] M. Oraldi, A. Trombetta, F. Biasi, R. A. Canuto, M. Maggiora, and G. Muzio, "Decreased polyunsaturated fatty acid content contributes to increased survival in human colon cancer," Journal of Oncology, vol. 2009, Article ID 867915, 9 pages, 2009.

[52] X. Y. Yang, L. H. Wang, and W. L. Farrar, "A role for PPAR $\gamma$ in the regulation of cytokines in immune cells and cancer," $P P A R$ Research, vol. 2008, Article ID 961753, 12 pages, 2008.

[53] A. H. V. Remels, R. C. J. Langen, H. R. Gosker et al., "PPAR $\gamma$ inhibits NF- $\kappa$ B-dependent transcriptional activation in skeletal muscle," American Journal of Physiology: Endocrinology and Metabolism, vol. 297, no. 1, pp. E174-E183, 2009.

[54] K. H. Weylandt, C.-Y. Chiu, B. Gomolka, S. F. Waechter, and B. Wiedenmann, "Omega-3 fatty acids and their lipid mediators: towards an understanding of resolvin and protectin formation," Prostaglandins and Other Lipid Mediators, vol. 97, no. 3-4, pp. 73-82, 2012.

[55] K. Mayer, A. Kiessling, J. Ott et al., "Acute lung injury is reduced in fat-1 mice endogenously synthesizing n-3 fatty acids," The American Journal of Respiratory and Critical Care Medicine, vol. 179, no. 6, pp. 474-483, 2009.

[56] B. Wang, X. Gong, J.-Y. Wan et al., "Resolvin D1 protects mice from LPS-induced acute lung injury," Pulmonary Pharmacology and Therapeutics, vol. 24, no. 4, pp. 434-441, 2011.

[57] K. G. Kreymann, M. M. Berger, N. E. P. Deutz et al., "ESPEN guidelines on enteral nutrition: intensive care," Clinical Nutrition, vol. 25, no. 2, pp. 210-223, 2006.

[58] P. Singer, M. M. Berger, G. van den Berghe et al., "ESPEN Guidelines on Parenteral Nutrition: intensive care," Clinical Nutrition, vol. 28, no. 4, pp. 387-400, 2009.

[59] S. A. McClave, R. G. Martindale, V. W. Vanek et al., "Guidelines for the provision and assessment of nutrition support therapy in the adult critically ill patient: society of critical care medicine (SCCM) and American society for parenteral and enteral nutrition (A.S.P.E.N.)," Journal of Parenteral and Enteral Nutrition, vol. 33, no. 3, pp. 277-316, 2009.

[60] Canadian clinical practice guidelines 2013 for nutrition support in mechanically ventilated, critically ill adult patients, http://www.criticalcarenutrition.com.

[61] S. Lev and P. Singer, "N-3 fatty acids and $\gamma$-linolenic acid supplementation in the nutritional support of ventilated patients with acute lung injury or acute respiratory distress syndrome," World Review of Nutrition and Dietetics, vol. 105, pp. 136-143, 2013. 

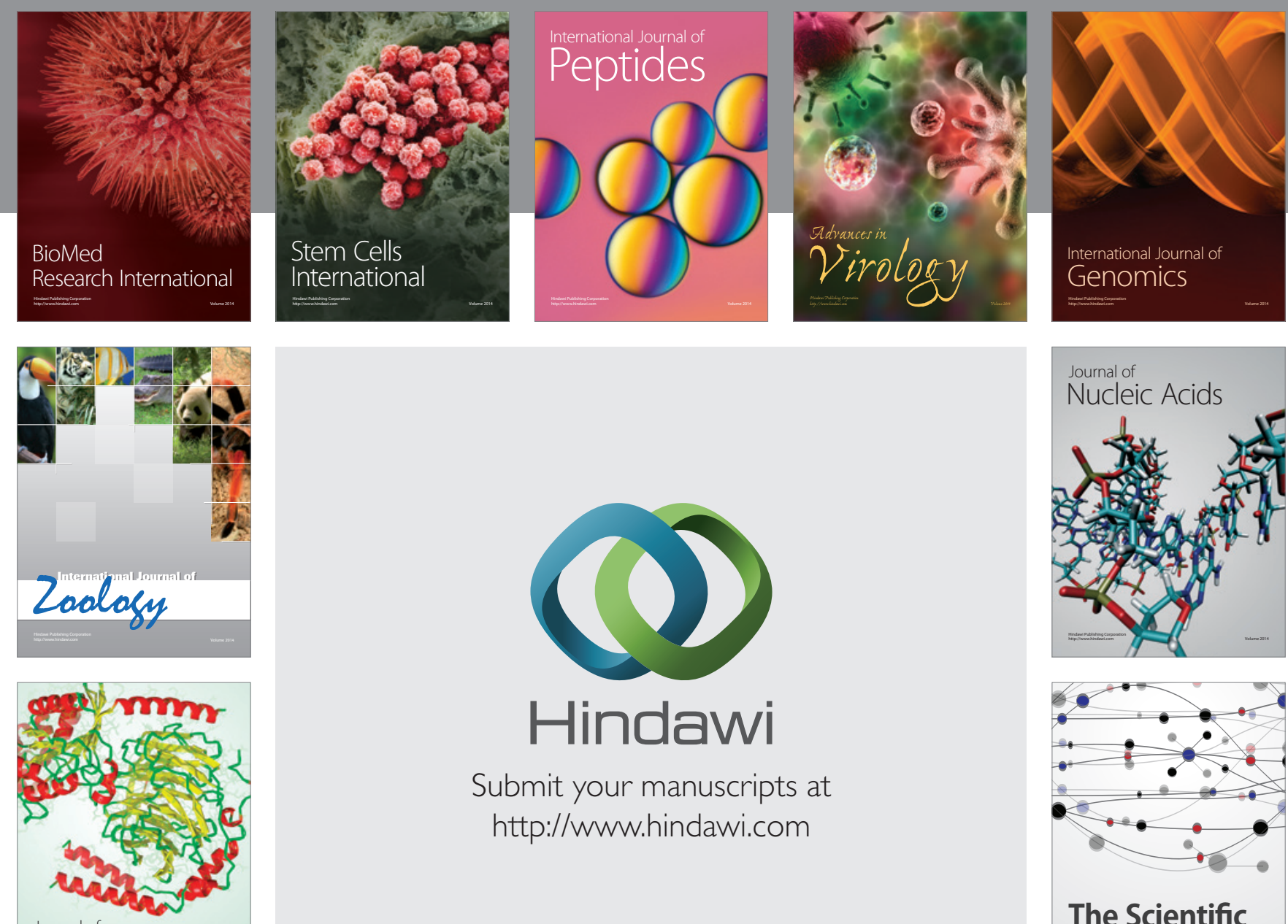

Submit your manuscripts at

http://www.hindawi.com

Journal of
Signal Transduction
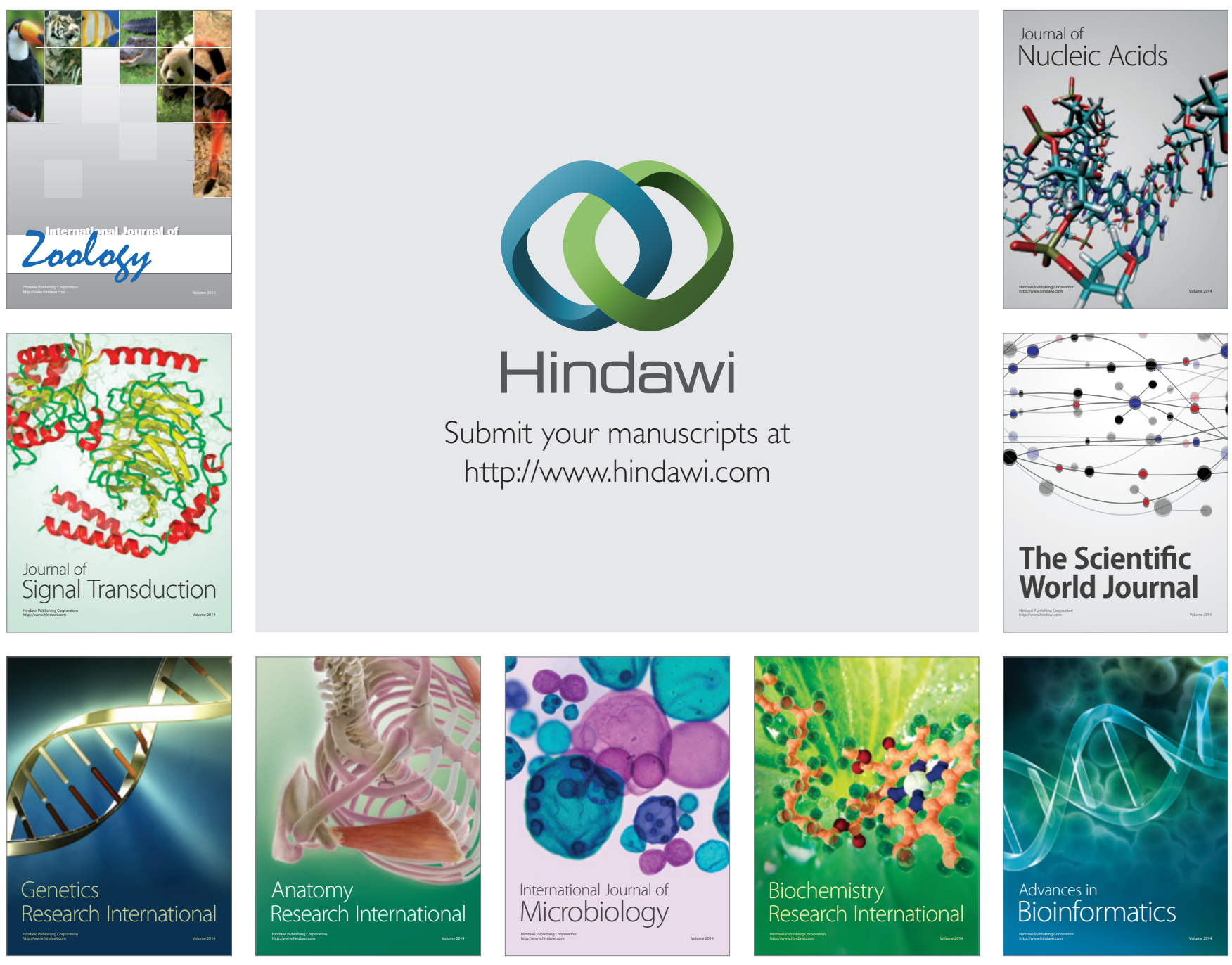

The Scientific World Journal
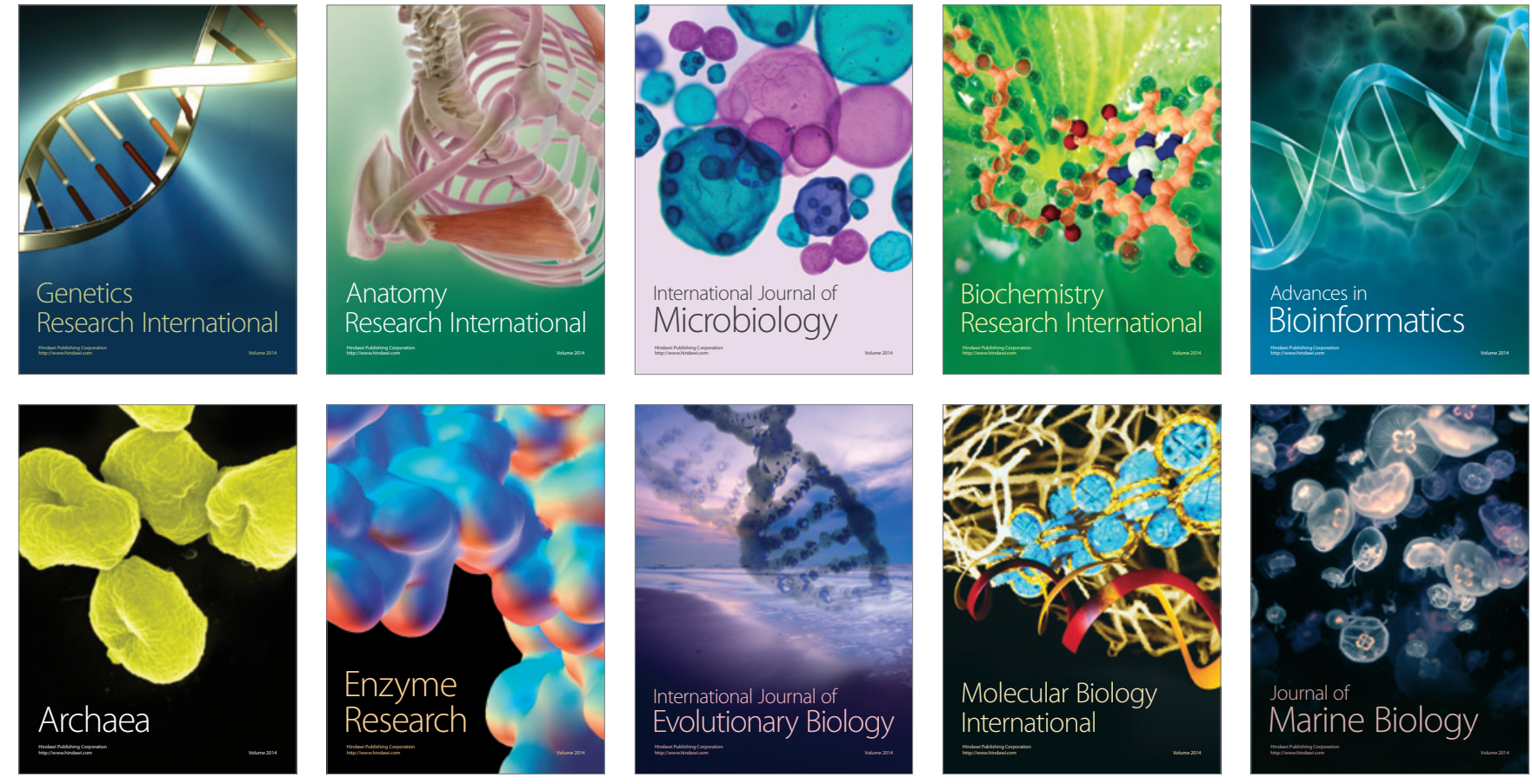